\title{
QUADRATIC PARTITIONS: PAPER IV
}

BY E. T. BELL

1. Identity of Degree 5. For the preceding note, see this Bulletin, vol. 38, p. 569 . The degree of a $\vartheta, \phi$ identity is the degree of the identity in functions $\vartheta, \phi$. In II we discussed an identity of degree 4 . Here we consider an identity of degree 5 whose equivalent in parity functions refers to $F(w, z, u, v)$ as in II. The identity is one of many of degree 5 by Gage.

Denote by $\Psi(w, z, u, v)$ the function

$$
\phi_{111}\left(\frac{w+z}{2}, u\right) \vartheta_{3}{ }^{2}\left(\frac{v-z}{2}\right) \vartheta_{3}{ }^{2}\left(\frac{w-u}{2}\right) \text {. }
$$

Then

$$
\begin{aligned}
\Psi(w, z, u, v)+ & \Psi(w,-z,-u,-v) \\
& -\Psi(w,-z, v, u)-\Psi(w, z,-v,-u) \equiv 0
\end{aligned}
$$

is an identity in $w, z, u, v$. The required expansions are

$$
\begin{aligned}
\vartheta_{3}(x) & =\sum q^{\nu^{2}} \cos 2 \nu x, \text { and } \\
\phi_{111}(x, y) & =\operatorname{ctn} x+\operatorname{ctn} y+4 \sum q^{2 n}\left[\sum \sin 2(d x+\delta y)\right] .
\end{aligned}
$$

For the notation in the above and in what follows, refer to $I$.

2. Equivalent of $\Psi$-Identity. To apply the formulas in I, $\$ 7$, to the reduction of the $\operatorname{ctn}$ terms, make the substitution $(w, z)$ $\rightarrow(x+y, x-y)$, and in the result apply

$$
(x, y) \rightarrow((w+z) / 2,(w-z) / 2) .
$$

Proceeding as in II, we find the following. The partitions are

$$
n=2 d \delta+\nu_{1}^{2}+\nu_{2}^{2}+\nu_{3}^{2}+\nu_{4}^{2}=a_{1}^{2}+{a_{2}}^{2}+{a_{3}}^{2}+a_{4}{ }^{2} .
$$

Write

$$
\begin{aligned}
\lambda_{1} & \equiv \nu_{1}+\nu_{2}, \lambda_{2} \equiv \nu_{3}+\nu_{4}, \alpha_{1} \equiv a_{1}+a_{2}, \alpha_{2} \equiv a_{3}+a_{4} \\
\sigma_{1} & \equiv \alpha_{1}+\alpha_{2}, \sigma_{2} \equiv \alpha_{1}-\alpha_{2}, \sigma_{1, r} \equiv \sigma_{2, r}+2 \sigma_{2}, \\
\sigma_{2, r} & \equiv 2 r-1+e(n)-\sigma_{2} ; S \equiv\left[\frac{1}{2}\left(\left|\sigma_{1}\right|-1\right)\right], A \equiv\left[\frac{1}{2}\left(\left|\alpha_{2}\right|-1\right)\right] .
\end{aligned}
$$

Then the identity gives 
698

$$
\begin{aligned}
& 4 \sum F\left(d+\lambda_{2}, d-\lambda_{1}, 2 \delta-\lambda_{2}, \lambda_{1}\right) \\
& =\sum \operatorname{sgn} \sigma_{1}\left[e(n) F\left(\sigma_{2} / 2, \sigma_{2} / 2, \alpha_{1}, \alpha_{2}\right)-F\left(\alpha_{2}, \alpha_{1}, \alpha_{1}, \alpha_{2}\right)\right. \\
& \left.\quad-2 \sum_{r=1}^{S} F\left(\sigma_{2, r} / 2, \sigma_{1, r} / 2, \alpha_{1}, \alpha_{2}\right)\right] \\
& \quad+\sum_{\operatorname{sgn} \alpha_{2}\left[e\left(\alpha_{2}\right) F\left(\alpha_{2}, \alpha_{1}, \alpha_{1}, 0\right)+F\left(\alpha_{2}, \alpha_{1}, \alpha_{1}, \alpha_{2}\right)\right.} \\
& \left.\quad+2 \sum_{r=1}^{A} F\left(\alpha_{2}, \alpha_{1}, \alpha_{1}, 2 r-1+e\left(\alpha_{2}\right)\right)\right],
\end{aligned}
$$

with $F$ as in II.

3. A Summation Formula. Let $f\left(x_{1}, \cdots, x_{t}\right)$ be entire in $\left(x_{1}, \cdots, x_{t}\right)$. Then

$$
\sum_{r=1}^{n} f\left(a_{1} r+b_{1}, \cdots, a_{t} r+b_{t}\right)=f\left(a_{1} \theta(n)+b_{1}, \cdots, a_{t} \theta(n)+b_{t}\right),
$$

where $\theta(n)$ is the umbra of $\theta_{j}(n)(j=0,1, \cdots)$ and $\theta_{j}(n) \equiv$ $\sum_{r=1}^{n} r^{j}$. The generators of $\theta(n), B$ are

Hence

$$
e^{\theta(n) x} \equiv \frac{e^{x}\left(e^{n x}-1\right)}{e^{x}-1}, \quad e^{B x} \equiv \frac{x}{e^{x}-1} .
$$

$$
x e^{\theta(n) x}=e^{(n+1+B) x}-e^{(1+B) x},
$$

and therefore, with $\theta_{s}(n) \equiv 0, s<0$,

$$
r \theta_{r-1}(n)=(n+1+B)^{r}-(1+B)^{r}, \quad(r=0,1, \cdots) .
$$

4. Application of $\S 3$ to $\S 2$. Denote by $F_{2}(w, z, u, v)$ the function $F(w, z, u, v)$ with the restriction of entirety in $(w, z, u, v)$. Then

$4 \sum F_{2}\left(d+\lambda_{2}, d-\lambda_{1}, 2 \delta-\lambda_{2}, \lambda_{1}\right)$

$$
\begin{aligned}
= & \sum \operatorname{sgn} \sigma_{1}\left[e(n) F_{2}\left(\sigma_{2} / 2, \sigma_{2} / 2, \alpha_{1}, \alpha_{2}\right)-F_{2}\left(\alpha_{2}, \alpha_{1}, \alpha_{1}, \alpha_{2}\right)\right. \\
& \left.-2 F_{2}\left(\theta(S)+\left(e(n)-1-\sigma_{2}\right) / 2, \theta(S)+\left(e(n)-1+\sigma_{2}\right) / 2, \alpha_{1}, \alpha_{2}\right)\right] \\
& +\sum \operatorname{sgn} \alpha_{2}\left[e\left(\alpha_{2}\right) F_{2}\left(\alpha_{2}, \alpha_{1}, \alpha_{1}, 0\right)+F_{2}\left(\alpha_{2}, \alpha_{1}, \alpha_{1}, \alpha_{2}\right)\right. \\
& \left.+2 F_{2}\left(\alpha_{2}, \alpha_{1}, \alpha_{1}, 2 \theta(A)+e\left(\alpha_{2}\right)-1\right)\right] .
\end{aligned}
$$

5. Contractions. We contract $F(w, z, u, v)$ with respect to $z$, as in II, $\S 6$. The new partitions are

$$
n=\sum_{i=1}^{4} \nu_{i}{ }^{2}-2 \delta^{2}=2 a_{1}{ }^{2}+a_{2}{ }^{2}+a_{3}{ }^{2}=\sum_{i=1}^{4} b_{i}{ }^{2},
$$


restricted as next stated. Write

$$
\begin{aligned}
& \lambda_{1} \equiv \nu_{1}+\nu_{2}, \lambda_{2} \equiv \nu_{3}+\nu_{4}, \alpha \equiv a_{2}+a_{3}, A \equiv\left[\frac{1}{2}(|\alpha|-1)\right] \\
& \beta_{1} \equiv b_{1}+b_{2}, \beta_{2} \equiv b_{3}+b_{4}, \sigma_{1} \equiv \beta_{1}+\beta_{2}, \sigma_{2} \equiv \beta_{1}-\beta_{2} .
\end{aligned}
$$

Then the restrictions are

$$
\lambda_{1}>2 \delta, e(n-1)>\sigma_{2} \geqq 2-\left|\sigma_{1}\right|, \sigma_{2} \equiv e(n-1) \bmod 2 .
$$

With $G, G_{1}$ as in II, we have

$$
\begin{aligned}
& 2 \sum G\left(\lambda_{1}+\lambda_{2}-2 \delta, 2 \delta-\lambda_{2}, \lambda_{1}-2 \delta\right) \\
& =\sum \operatorname{sgn} \alpha\left[\sum_{r=1}^{A} G(\alpha, 0,2 r-e(n-1))\right] \\
& \quad+\sum \operatorname{sgn} \sigma_{1} G\left(\sigma_{2}, \beta_{1}, \beta_{2}\right) ; \\
& 2 \sum G_{1}\left(\lambda_{1}+\lambda_{2}-2 \delta, 2 \delta-\lambda_{2}, \lambda_{1}-2 \delta\right) \\
& =\sum \operatorname{sgn} \alpha G_{1}(\alpha, 0,2 \theta(A-e(n-1)) \\
& \quad+\sum \operatorname{sgn} \sigma_{1} G_{1}\left(\sigma_{2}, \beta_{1}, \beta_{2}\right) .
\end{aligned}
$$

In the reductions we have used

$$
\begin{aligned}
1-e(\nu) & =e(\nu-1), \\
2\left[\frac{1}{2}(|\nu|-1)\right] & =|\nu|-1-e(\nu), \sigma_{1} \equiv n \bmod 2 .
\end{aligned}
$$

Alternative forms are obtained on noticing that

$$
\operatorname{sgn} w F(w, z, u, v)=F(|w|, z, u, v) .
$$

California Institute of Technology 